Alberta Thy 02-03

\title{
Optimal Jet Finder
}

\author{
D. Yu. Grigoriev \\ Mathematical Physics, Natl. Univ. of Ireland Maynooth, Maynooth, Co. Kildare, \\ Ireland and Institute for Nuclear Research of RAS, Moscow 117312, Russia \\ E. Jankowski \\ Department of Physics, University of Alberta, Edmonton, AB, T6G 2J1, Canada \\ F. V. Tkachov ${ }^{1}$ \\ Institute for Nuclear Research of RAS, Moscow 117312, Russia
}

\begin{abstract}
We describe a FORTRAN 77 implementation of the optimal jet definition for identification of jets in hadronic final states of particle collisions. We discuss details of the implementation, explain interface subroutines and provide a usage example. The source code is available from http://www.inr.ac.ru/ ftkachov/projects/jets/
\end{abstract}

Keywords: hadronic jets, jet finding algorithms

PACS: 13.87.-a, 29.85.+c

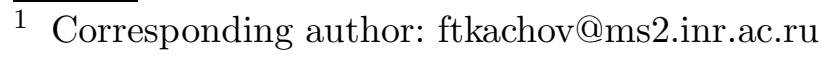

Preprint submitted to Elsevier Science

30 October 2018 


\section{PROGRAM SUMMARY}

Title of program: Optimal Jet Finder (OJF_014)

Catalogue identifier: (supplied by the Publisher)

Distribution format: (supplied by the Program Library)

Computer: any computer with the FORTRAN 77 compiler

Tested with: g77/Linux on Intel, Alpha and Sparc; Sun f77/Solaris (thwgs.cern.ch); xlf/AIX (rsplus.cern.ch); MS Fortran PowerStation 4.0/Win98

Programming language used: FORTRAN 77

Memory required: $\sim 1 \mathrm{MB}$ (or more, depending on the settings)

Number of bytes in distributed program, including examples and test data: $1.39 \mathrm{MB}$

Keywords: hadronic jets; jet finding algorithms

Nature of physical problem

Analysis of hadronic final states in high energy particle collision experiments often involves identification of hadronic jets. A large number of hadrons observed in the detector is reduced to a few jets by means of a jet finding algorithm. The jets are used in further analysis which would be difficult or impossible when applied directly to the hadrons. Reference [1] provides a brief introduction to the subject of jet finding algorithms and a general review of the physics of jets can be found in [2].

\section{Method of solution}

The software we provide is an implementation of the so-called optimal jet definition (OJD). The theory of OJD was developed in [3], [4], [5]. The desired jet configuration is obtained as the one that minimizes $\Omega_{R}$, a certain function of the input particles and jet configuration.

Restrictions on the complexity of the program

The size of the largest data structure the program uses is (maximal number of particles in the input) $\times($ maximal number of jets in the output) $\times 8$ bytes. (For the standard settings $<1 \mathrm{MB}$ ). Therefore, there is no memory restriction for any conceivable application for which the program was designed.

\section{Typical running time}

The running time depends strongly on the physical process being analyzed and the parameters used. For the benchmark process we studied, $\mathrm{e}^{+} \mathrm{e}^{-} \rightarrow \mathrm{W}^{+} \mathrm{W}^{-} \rightarrow 4$ jets, with the average number of $\sim 80$ particles in the input, the running time was 
$<10^{-2} \mathrm{~s}$ on a modest PC per event with $n_{\text {tries }}=1$. (We took $n_{\text {tries }}=10$ for all events in the benchmark process, however for the majority of events $n_{\text {tries }} \sim 3$ would suffice. For small values of $n_{\text {tries }}$ the global minimum of $\Omega_{R}$ may be missed in some fraction of events resulting in the deterioration of the precision of measurements based on the jet algorithm.) For a fixed number of jets the complexity of the algorithm grows linearly with the number of particles (cells) in the input, in contrast with other known jet finding algorithms for which this dependence is cubic. The reader is referred to [1] for a more detailed discussion of this issue.

\section{References}

[1] D. Yu. Grigoriev, E. Jankowski, F. V. Tkachov, e-print hep-ph/0301185, to be published.

[2] R. Barlow, Rep. Prog. Phys. 36, 1067 (1993).

[3] F. V. Tkachov, Phys. Rev. Lett. 73, 2405 (1994); Erratum, 74, 2618 (1995).

[4] F. V. Tkachov, Int. J. Mod. Phys. A12, 5411 (1997).

[5] F. V. Tkachov, Int. J. Mod. Phys. A17, 2783 (2002). 


\section{Contents}

$1 \quad$ Introduction 5

\begin{tabular}{lll}
\hline 2 & Algorithm & 8
\end{tabular}

2.1 Criterion for the optimal jet configuration 8

$\begin{array}{lll}2.2 \text { Algorithm for minimizing } \Omega & 10\end{array}$

\begin{tabular}{lll}
\hline 2.3 & Formulas & 12
\end{tabular}

$\begin{array}{lll}3 \quad \text { Code and usage } & 12\end{array}$

$\begin{array}{lll}3.1 & \text { Code and data structure } & 13\end{array}$

$\begin{array}{lll}3.2 & \text { Normalization of energv units } & 14\end{array}$

$\begin{array}{lll}3.3 & \text { Error messages } & 14\end{array}$

\begin{tabular}{|ll}
$3.4 \quad$ Kev minimization subroutine Q_minimize & 14
\end{tabular}

$\begin{array}{lll}3.5 & \text { User callable subroutines } & 15\end{array}$

\begin{tabular}{|ll}
\hline 3.6 & Compilation \\
\hline
\end{tabular}

\begin{tabular}{|ll}
3.7 & Example
\end{tabular}

4 Definitions of constants: ojf_par.fh 30

5 Common block definitions: ojf_com.fh 32

$5.1 \quad$ Input of the event 32

5.2 Configuration of jets (output) 33

\begin{tabular}{|lll}
$6 \quad$ Conclusion & 35
\end{tabular}

\begin{tabular}{ll}
\hline References & 35
\end{tabular} 


\section{Introduction}

This paper represents an official public release of the Optimal Jet Finder (OJF) library - a FORTRAN 77 implementation of the so-called optimal jet definition (OJD) for identification of jets in hadronic final states of particle collisions. For a brief introduction into the subject the reader is referred to [1], and a general review of the physics of jets can be found in [2]. The theory of OJD was developed in [3], [4], [5], to which papers the reader is referred for a detailed discussion of the physical motivations behind and the derivation of OJD. The principal points of the theory are summarized below.

(i) Calorimetric measurements with hadronic final states $\mathbf{P}$ must rely on observables $f(\mathbf{P})$ that possess a special "calorimetric", or $C$-continuity which is a non-perturbative generalization of the familiar IR safety (see [4] for details) and which guarantees a stability of $f(\mathbf{P})$ against distortions of $\mathbf{P}$ such as caused by detectors. Ref. [4] pointed out $C$-continuous analogues for a variety of observables usually studied via intermediacy of jet algorithms. The fundamental role of such observables is highlighted by two facts: (1) An observable inspired by [4] played an important role in the selection of top quark events in the fully hadronic channel at D0 [8], [9]. (2) The Jet Energy Flow project [10] provides numerical evidence that $C$-continuous observables may indeed help to go beyond the intrinsic limitations of conventional procedure based on jet algorithms in the quest for the $1 \%$ precision level in the physics of jets.

(ii) The proposition that the observed event $\mathbf{P}$ inherits information (as measured by calorimetric detectors) from the underlying quark-and-gluon event $\mathbf{q}$ is expressed as

$$
f(\mathbf{q}) \approx f(\mathbf{P}) \quad \text { for any } C \text {-continuous } f .
$$

(iii) For each parameter $M$ on which the probability distribution $\pi_{M}(\mathbf{P})$ of the observed events $\mathbf{P}$ may depend, there exists an optimal observable $f_{\text {opt }}(\mathbf{P})=\partial_{M} \ln \pi_{M}(\mathbf{P})$ for the best possible measurement of $M$ [7]. This is a reinterpretation of the Rao-Cramer inequality and the maximal likelihood method of mathematical statistics in terms of the method of moments. In the context of multi-hadron final states as "seen" by calorimetric detectors, such an observable is automatically $C$-continuous.

(iv) If the dynamics of hadronization is such that eq. (1) holds, then good approximations for $f_{\text {opt }}$ could exist among functions that depend only on $\mathbf{Q}$ which is a parameterization of $\mathbf{P}$ in terms of a few pseudo-particles (jets), found from a condition modeled after eq. (1):

$$
f(\mathbf{Q}) \approx f(\mathbf{P}) \quad \text { for any } C \text {-continuous } f \text {. }
$$


This simply translates the meaning of jet finding as an inversion of hadronization into the language of $C$-continuous observables.

(v) $C$-continuous observables can be approximated by sums of products of simplest such observables that are linear in particles' energies $E_{a}$ :

$$
f(\mathbf{P})=\sum_{a} E_{a} f\left(\hat{\mathbf{p}}_{a}\right),
$$

where $a$ runs over all particles in the event and $\hat{\mathbf{p}}_{a}$ (a unit vector) denotes the direction of the 3-momentum of the $a$-th particle; $f$ is any continuous function of a direction only. (The relevant theorems can be found in refs. [4] and [5].)

(vi) So it is sufficient to explore the criterion (2) with only $f$ 's of the form (3). Then one can perform a Taylor expansion in angular variables and obtain a factorized bound of the form

$$
|f(\mathbf{P})-f(\mathbf{Q})| \leq C_{f, R} \times \Omega_{R}[\mathbf{P}, \mathbf{Q}],
$$

where all the dependence on $f$ is localized within $C_{f, R}$ (so the bound remains valid for any $C$-continuous $f$ ) and $\Omega_{R}[\mathbf{P}, \mathbf{Q}]$ is a function of the jet configuration $\mathbf{Q}$ (and the event $\mathbf{P}$ ) only (the explicit form is given in section 2.1).

(vii) Since the collection of values of all $f$ on a given event $\mathbf{P}$ is naturally interpreted as the event's physical information content, the bound (4) means that the distortion of such content in the transition from $\mathbf{P}$ to $\mathbf{Q}$ can be controlled by a single function; so the loss of physical information in the transition is minimized if $\mathbf{Q}$ corresponds to the global minimum of $\Omega_{R}$. The Optimal Jet Definition amounts to finding the jet configuration $\mathbf{Q}$ which minimizes $\Omega_{R}[\mathbf{P}, \mathbf{Q}]$, depending on specific application, either with a given number of jets or with a minimum number of jets while satisfying the restriction $\Omega_{R}[\mathbf{P}, \mathbf{Q}]<\omega_{\text {cut }}$ with some parameter $\omega_{\text {cut }}>0$ which is similar to the jet resolution $y_{\text {cut }}$ of recombination algorithms.

The beta versions of the OJF library have been available since 1999 (see [6]). The publicly available code [11] was first developed in the programming language Component Pascal [12] from the highly regarded Pascal/Modula2/Oberon family, featuring a unique combination of intellectual manageability, safety, and efficiency of compiled code (for more on this see [13]). This made possible the experimentation needed to find a working and correct algorithm. Only after that the final port to FORTRAN 77 was performed. A subsequent testing on a total of $O\left(10^{10}\right)$ events [14] and a substantially independent verification [15] revealed no defects of significance, indicating a high reliability of the code as a result of the adopted development process.

The OJF library can be used to obtain OJD implementations adapted for 
specific applications (see below).

A typical program based on OJF takes as an input the collection of particles (or detector cells), characterizing them by energies and directions. Then the optimal jet configuration is found by minimizing some function of the particles and jet configuration. Each particle can be shared among jets (in conventional schemes only an entire particle can belong to a single jet), so for an input event containing $n$ particles and resulting in $N$ jets, the number of degrees of freedom is $n \cdot N$. A typical application (for instance, analysis of data for LHC) may require $n \sim 100 \ldots 400$ and $N \sim 6$ and therefore $n \cdot N \sim O(1000)$. A large number of degrees of freedom makes the minimization problem non-trivial. The program we describe implements a practical algorithm (taking a fraction of a second per event on a modest personal computer) solving the optimization problem inherent in OJD. A key subroutine performing the minimization is Q_minimize.

For a fixed number of jets the computational complexity of the algorithm grows as the first power of the number of particles in the input, $O(n)$ (see [1] for the corresponding empirical data; for comparison, the computational complexity is $O\left(n^{3}\right)$ for the kT algorithm [16], [17].) This feature makes OJF especially attractive for processing events with many calorimetric cells in the input. For a benchmark process we studied, $\mathrm{e}^{+} \mathrm{e}^{-} \rightarrow \mathrm{W}^{+} \mathrm{W}^{-} \rightarrow 4$ jets, OJF started to become more time efficient than the standard kT roughly at 90 cells in the input. ${ }^{2}$

The version of OJF being described is called OJF_014. The FORTRAN 77 source code is available from http://www.inr.ac.ru/ ftkachov/projects/jets/

OJF contains many interface subroutines (usually with names starting with set_or get_) allowing to input data, read output and change the algorithm parameters. The user is not supposed to write data directly to common blocks but is to use the interface subroutines. Similarly, only the parameters that can be accessed by the user callable subroutines are supposed to be changed (at least at level of standard application).

OJF has been compiled on various platforms: g77/Linux on Intel, Alpha and Sparc, Sun f77/Solaris (thwgs.cern.ch), xlf/AIX (rsplus.cern.ch), MS Fortran PowerStation 4.0/Win98. Its numerical stability was tested on a total of $\sim 10^{10}$ events.

OJF contains options to handle both the center of mass (spherical) kinematics of lepton collisions and the cylindrical kinematics of hadron collisions.

${\overline{2} \text { For } n_{\text {ntries }}}=1$, see section 2.1 . 


\section{Algorithm}

\subsection{Criterion for the optimal jet configuration}

The input for the program is an event: a collection of $n$ particles from the detector (or $n$ hit detector cells), indexed with $a=1,2,3, \ldots, n$. Each particle is characterized by its energy, $E_{a}$, and its direction described by the standard angles $\theta_{a}, \varphi_{a}$ or equivalently by transverse energy, $E_{a}^{\perp}$, pseudorapidity, $\eta_{a}$, and the angle $\varphi_{a}$. The $a$-th particle in the input is assigned the 4-momentum $p_{a}$ :

$$
p_{a}=E_{a} \cdot\left(1, \sin \theta_{a} \cos \varphi_{a}, \sin \theta_{a} \sin \varphi_{a}, \cos \theta_{a}\right)
$$

or

$$
p_{a}=E_{a}^{\perp} \cdot\left(\cosh \eta_{a}, \cos \varphi_{a}, \sin \varphi_{a}, \sinh \eta_{a}\right) .
$$

depending on what parameters describe the particles. The output of the program is a set of $N$ jets, indexed with $j=1,2,3, \ldots, N$. The jet configuration is described by recombination matrix $\left\{z_{a j}\right\}$ components of which satisfy:

$$
\begin{aligned}
& 0 \leq z_{a j} \leq 1 \quad \text { for } \text { all } a, j \\
& \sum_{j=1}^{N} z_{a j} \leq 1 \quad \text { for all } a .
\end{aligned}
$$

The number $z_{a j}$ gives the fraction of the $a$-th particle which goes into formation of the $j$-th jet. Each $z_{a j}$ can take any value between 0 and 1 . The final value of the recombination matrix $\left\{z_{a j}\right\}$ is the result of the algorithm. The 4 -momentum $q_{j}$ of the $j$-th jet is defined as

$$
q_{j}=\sum_{a=1}^{n} z_{a j} p_{a} .
$$

The final (optimal) jet configuration is the one that minimizes the value of some function $\Omega\left(\left\{z_{a j}\right\}\right)$ depending on the recombination matrix $\left\{z_{a j}\right\}$ and all $p_{a}$ as parameters.

The definition of $\Omega$ follows some intermediate sub-definitions. Part of the $a$-th particle that do not go into formation of any jet:

$$
\bar{z}_{a} \equiv 1-\sum_{j=1}^{N} z_{a j} .
$$


The rest of the definitions are given separately for spherical kinematics (lepton collisions) and for cylindrical kinematics (hadron collisions).

Spherical kinematics. Overall energy left outside jets $E_{\text {soft }}$, called soft energy:

$$
E_{\mathrm{soft}} \equiv \sum_{a=1}^{n} \bar{z}_{a} E_{a}
$$

The function $Y$, called fuzziness:

$$
Y \equiv 2 \sum_{j=1}^{N} q_{j} \widetilde{q}_{j}
$$

where $\widetilde{q_{j}}$ is light-like $\left({\widetilde{q_{j}}}^{2}=0\right)$ 4-direction defined:

$$
\widetilde{q_{j}} \equiv\left(1, \sin \theta_{j} \cos \varphi_{j}, \sin \theta_{j} \sin \varphi_{j}, \cos \theta_{j}\right)
$$

with

$$
\begin{aligned}
\cos \theta_{j} & \equiv \frac{\left(q_{j}\right)_{z}}{\sqrt{\left(q_{j}\right)_{x}^{2}+\left(q_{j}\right)_{y}^{2}+\left(q_{j}\right)_{z}^{2}}} \\
\cos \varphi_{j} & \equiv \frac{\left(q_{j}\right)_{x}}{\sqrt{\left(q_{j}\right)_{x}^{2}+\left(q_{j}\right)_{y}^{2}}}, \\
\sin \varphi_{j} & \equiv \frac{\left(q_{j}\right)_{y}}{\sqrt{\left(q_{j}\right)_{x}^{2}+\left(q_{j}\right)_{y}^{2}}} .
\end{aligned}
$$

Cylindrical kinematics. The soft energy is the overall transverse energy left outside the jets

$$
E_{\text {soft }} \equiv \sum_{a=1}^{n} \bar{z}_{a} E_{a}^{\perp} \text {. }
$$

The fuzziness $Y$ is defined again by (12) with $\widetilde{q}_{j}$, light-like $\left(\widetilde{q}_{j}^{2}=0\right)$ 4-direction given by:

$$
\widetilde{q_{j}} \equiv\left(\cosh \eta_{j}, \cos \varphi_{j}, \sin \varphi_{j}, \sinh \eta_{j}\right)
$$


where

$$
\eta_{j} \equiv \frac{\sum_{a=1}^{n} z_{a j} E_{a}^{\perp} \eta_{a}}{\sum_{a=1}^{n} z_{a j} E_{a}^{\perp}}
$$

and $\cos \varphi_{j}, \sin \varphi_{j}$ given by (15), (16).

Finally, in both cases, $\Omega$ is a linear combination of $Y$ and $E_{\text {soft }}$ with the parameter $R$ weighting their relative contribution:

$$
\Omega\left(\left\{z_{a j}\right\}\right) \equiv \frac{1}{R^{2}} Y+E_{\text {soft }} .
$$

If we intend to reconstruct an event to some fixed number of jets the procedure is as follows. Start with some initial value of the recombination matrix $z_{a j}$, for example chosen randomly and minimize the function $\Omega\left(\left\{z_{a j}\right\}\right)$ with respect to $\left\{z_{a j}\right\}$. The algorithm described in section 2.2 allows one to find a local minimum while starting with some initial value of $\left\{z_{a j}\right\}$. Repeat the procedure a few times (parameter $n_{\text {tries }}$ ), starting each time with different initial value of $\left\{z_{a j}\right\}$ and take the smallest of the minima obtained at each try. The value of the recombination matrix $\left\{z_{a j}\right\}$ that gives the smallest of the minima is the final jet configuration. If the initial value of $\left\{z_{a j}\right\}$ is not chosen randomly it is useless to do the minimization procedure more than once as the minimization algorithm is deterministic.

If the number of jets is to be determined in the process of jet reconstruction we can repeat the procedure described above for different number of jets $N$ each time. This means, we start with some minimal number of jets, e.g. $N=1$ and find the corresponding $N$-jet configuration and check whether

$$
\Omega\left(\left\{z_{a j}\right\}\right)<\omega_{\text {cut }}
$$

is fulfilled for that configuration. If so, this is the final jet configuration we look for. If not, we increase $N$ by one and repeat the $N$-jet procedure for the new $N$. We continue until (21) is finally satisfied (which has to be true for some sufficiently large number of jets). The parameter $\omega_{\text {cut }}$ is some (small) positive number one chooses and it is analogous to the jet resolution parameter of conventional recombination algorithms.

\subsection{Algorithm for minimizing $\Omega$}

The domain of the function $\Omega\left(\left\{z_{a j}\right\}\right)$ is a $(n \cdot N)$-dimensional product of simplices. That is, for fixed $a$ the numbers $z_{a j}, j=1, \ldots, N$, satisfying conditions 
(7), (8) define an $N$-dimensional simplex. In typical application $n \sim 200$ (or more) and $N \sim 5$ and therefore $\Omega$ is a function of $\sim 1000$ variables. The algorithm described below allows for efficient minimization of $\Omega\left(\left\{z_{a j}\right\}\right)$.

The algorithm iteratively descends into local minimum of $\Omega\left(\left\{z_{a j}\right\}\right)$ starting from a given initial value of $\left\{z_{a j}\right\}$. At each iteration, subsequently for each particle, $\left\{z_{a j}\right\}$ is moved into new position in the domain that gives the smaller value of $\Omega$. The iteration loop is terminated when no particle is moved at a single iteration, meaning that the local minimum has been found. (Or some safe number of maximal iterations has been exceeded.)

We describe now in detail how $\left\{z_{a j}\right\}$ is moved in a single iteration step for a given particle. Denote $\mathbf{z} \equiv\left(z_{1}, z_{2}, \ldots, z_{N}\right)$ with $z_{j}=z_{a j}$ and $\Omega(\mathbf{z}) \equiv \Omega\left(\left\{z_{a j}\right\}\right)$ with fixed $a$ in both definitions. The change in $\Omega$ when we change $\mathbf{z}$ to $\mathbf{z}+\tau \mathbf{d}$ can be described locally as

$$
\Omega(\mathbf{z}+\tau \mathbf{d})=\Omega(\mathbf{z})+\tau \mathbf{f} \cdot \mathbf{d}+O\left(\tau^{2}\right),
$$

where $\mathbf{f}=\left(f_{1}, \ldots, f_{N}\right), f_{j} \equiv \partial \Omega(\mathbf{z}) / \partial z_{j}, \mathbf{f} \cdot \mathbf{d}=\sum_{j=1}^{N} f_{j} d_{j}$ and $\mathbf{d}=\left(d_{1}, \ldots, d_{N}\right)$ describes some direction. If $\mathbf{z}$ were not constrained to the simplex we could take $\mathbf{d}=-\mathbf{f}$ and some $\tau>0$ to decrease $\Omega$. But choosing $\tau$ and $\mathbf{d}$ we have to ensure that $\mathbf{z}+\tau \mathbf{d}$ is within the simplex. Rewrite

$$
\mathbf{f} \cdot \mathbf{d}=\sum_{j=1}^{N} f_{j} d_{j}=\sum_{j=1}^{N} \bar{f}_{j} d_{j}+\bar{f}_{0} d_{0}
$$

with the following definitions

$$
\begin{aligned}
\bar{f}_{j} & \equiv f_{j}-f_{J}, \quad \bar{f}_{0} \equiv-f_{J}, \\
d_{0} & \equiv-\sum_{j=1}^{N} d_{j},
\end{aligned}
$$

where $J$ is any of $1, \ldots, N$ for which $z_{J}>0$ (there always must be such $J$ ). Now $\mathbf{d}$ can be chosen as follows

$$
d_{j} \equiv \begin{cases}\max \left(0,-\bar{f}_{j}\right) & \text { for all } j=0, \ldots, N, \text { for which } z_{j}=0 \\ -\bar{f}_{j} & \text { for all } j=0, \ldots, N, j \neq J \text { for which } z_{j}>0\end{cases}
$$

and $d_{J}$ is chosen so that (25) is satisfied. With such choice of $\mathbf{d}$ and the proper parameter $\tau$ the new candidate minimum $\mathbf{z}+\tau \mathbf{d}$ will belong to the simplex 
and $\Omega(\mathbf{z}+\tau \mathbf{d})<\Omega(\mathbf{z})$. In the above prescription the choice of $J$ is arbitrary. We found it advantageous to choose $J\left(z_{J}>0\right)$ such that the norm

$$
\left|\left(\mathbf{d}, d_{0}\right)\right| \equiv \max \left\{\left|d_{j}\right|: j=0,1, \ldots, N\right\}
$$

is maximal. The choice of step length $\tau$ is determined by the experimental finding that the minimum tends to be located at the boundary of the simplex. We find

$$
\tau=\min \left(\left\{-\frac{z_{j}}{d_{j}}: \quad j=0, \ldots, N, z_{j}>0 \text { and } d_{j}<0\right\}\right)
$$

from the requirement that the new candidate minimum $\mathbf{z}+\tau \mathbf{d}$ should be located at the boundary of the simplex and if this results in an increase of the value of $\Omega$ then $\tau$ is iteratively divided by a constant factor $(\sim 3)$ until minimum is found.

An important technical implementation detail is the so-called "snapping". If some $z_{a j}$ is small enough (i.e. $\mathbf{z}$ is close enough to a boundary of the simplex) then it is set to zero. A similar snapping is used for the direction $\mathbf{d}$.

\subsection{Formulas}

We give here the explicit formulas for derivatives $f_{j} \equiv \partial \Omega\left(\left\{z_{a j}\right\}\right) / \partial z_{a j}$ used within the algorithm (derived from the definitions given in the previous sections).

\section{Spherical kinematics:}

$$
f_{j}=2 p_{a} \tilde{q}_{j}-E_{a} .
$$

\section{Cylindrical kinematics:}

$$
f_{j}=2 p_{a} \tilde{q}_{j}-\frac{E_{a}^{\perp}}{\sum_{j=1}^{N} z_{a j} E_{a}^{\perp}}\left(\eta_{a}-\eta_{j}\right)\left(q_{j}^{0} \sinh \eta_{j}-q_{j}^{\mathrm{z}} \cosh \eta_{j}\right)-E_{a}^{\perp} .
$$

\section{Code and usage}

We describe a FORTRAN 77 implementation of the algorithm explained in the previous section. 


\subsection{Code and data structure}

The code (file ojf_014.f) consists of subroutines (and functions) which can be divided in three logical groups: (i) interface subroutines, (ii) core subroutines and (iii) example jet search or result printing subroutines. In addition block data ojf_lock contains default values of some program parameters. The interface subroutines allow the user to enter input data, to read output or already entered input, to set or change program parameters and to obtain information about current program parameters. All parameters that are supposed to be set or changed by the user can be accessed by these subroutines. The same applies to all input and output data. The user is not supposed to write directly to common blocks. The core subroutines (functions) perform $\Omega\left(\left\{z_{a j}\right\}\right)$ minimization and conversion between various data forms. The user is not supposed to call them directly except for Q_minimize. The subroutine Q_search is an example application of OJF frame to simple jet search (see section 3.5.7). The user may want to modify it or write their own subroutines if needed.

All floating point variables within the program are defined as DOUBLE PRECISION. If the user employs REAL type variables they should ensure that a proper conversion of the parameter values is made in the calls of the OJF subroutines.

The file ojf_com. fh contains common block definitions of internal data structures for OJF, for instance, matrices for parameters of input particles, output jets parameters and recombination matrix $\left\{z_{a j}\right\}$. The file ojf_par.fh contains the definitions of constants used within the program. The file ojf_kin.fh contains the definitions of two constants: sphere=1, cylinder=2. The file can be contained in user programs whenever reference to kinematics type is made, e.g.

..

INCLUDE 'ojf_kin.fh'

INTEGER kinematics

...

kinematics $=$ sphere

event_setup_begin( kinematics )

...

The other two files (ojf_com.fh and ojf_kin.fh) normally do not need to be contained in user programs. 


\subsection{Normalization of energy units}

The energies $E_{a}$ or $E_{a}^{\perp}$ of input particles and the corresponding 4-momenta $p_{a}$ are normalized (after being entered) according to

$$
E_{a} \rightarrow \frac{E_{a}}{\sum_{a=1}^{n} E_{a}}, \quad p_{a} \rightarrow \frac{p_{a}}{\sum_{a=1}^{n} E_{a}}
$$

for spherical kinematics or according to

$$
E_{a}^{\perp} \rightarrow \frac{E_{a}^{\perp}}{\sum_{a=1}^{n} E_{a}^{\perp}}, \quad p_{a} \rightarrow \frac{p_{a}}{\sum_{a=1}^{n} E_{a}^{\perp}}
$$

for cylindrical kinematics. The normalization constant $\sum_{a=1}^{n} E_{a}$ or $\sum_{a=1}^{n} E_{a}^{\perp}$ is stored to interpret properly the final output. The normalization allows to make the implementation independent of energy units and scale.

\subsection{Error messages}

Significant part of the code consists of various checks. For example:

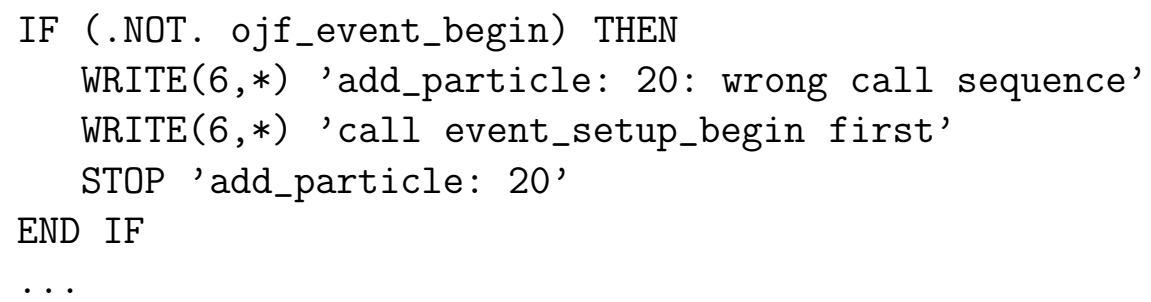

The checks are used to assure that subroutines are not called in inappropriate order, chosen parameters or input data do not have pathological values and that the program runs properly. The check can generate an error message and terminate the program. Messages with numbers 20-29 are due to the user errors. Messages with numbers $\geq 30$ are generated by program failures, so should you get such a message, please inform the authors; please include the corresponding event in text form.

\subsection{Key minimization subroutine Q_minimize}

The subroutine Q_minimize minimizes $\Omega\left(\left\{z_{a j}\right\}\right)$ for a given number of jets starting from the existing configuration of $\left\{z_{a j}\right\}$. An example program that 
uses Qminimize is given in section 3.7.

The subroutine performs iteratively the minimization algorithm described in section 2.2. The iteration loop is terminated when no particle is moved in a single iteration or the maximal number of iterations is exceeded. (We regard that the minimum is found only in the former case.) Default value of the maximal number of iterations is set 1000 which corresponds to $\sim 1$ second of computing time on a modest computer. It can be changed with set_maxiter ( maxiter ), see section 3.5.3. In each iteration, a loop over all particles is run $(a=1,2, \ldots, n)$. For each particle separately, new candidate $\left\{z_{a j}\right\}$ for the minimum is found. The direction, $\mathbf{d}$, and step , $\tau$, are computed according to the procedure described in section 2.2. Unless the step is zero or "infinity", indicating that the particle should not be moved, the condition

$$
\Omega(\mathbf{z}+\tau \mathbf{d})<\Omega(\mathbf{z})
$$

is checked. If the condition is met the recombination matrix $\left\{z_{a j}\right\}$ is moved into the new position. If not, the step is reduced 3 times and (33) is checked again. If it is not true $\tau$ is reduced again and so on. If $\tau$ falls below some small parameter $\left(\tau\left|\left(\mathbf{d}, d_{0}\right)\right| \leq\right.$ eps_dist $)$ the particle is not moved and the program proceeds to the next particle.

\subsection{User callable subroutines}

We describe all user callable subroutines other than Q_minimize explained above.

\subsubsection{Event setup}

event_setup_begin ( kinematics )

input:

INTEGER kinematics kinematics type

The subroutine begins initialization of a new event. It must be called before event data is entered. The parameter kinematics informs the program what type of kinematics is used: spherical (center of mass collisions), kinematics $=1$ or cylindrical (hadron collisions), kinematics $=2$. If the file ojf_kin.fh is included constants sphere and cylinder can be used to assign value to kinematics:

INCLUDE 'ojf_kin.fh' 
INTEGER kinematics

...

kinematics $=$ sphere

event_setup_begin( kinematics )

...

Kinematics ought to be set once for all events in a job.

add_particle ( energy, theta, phi )
input:
DOUBLE PRECISION energy energy $E_{a}$
DOUBLE PRECISION theta angle $\theta_{a}$
DOUBLE PRECISION phi angle $\phi_{a}$

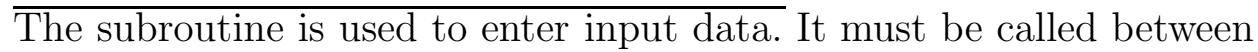
event_setup_begin and event_setup_end. Each call adds a particle (=detector cell) to the event. The energy $E_{a}$ of the particle can be in any units, for example $\mathrm{GeV}$. The direction of the particle is described by the standard angles $\theta_{a}$ (measured from beam axis) and $\phi_{a}$.

add_particle_raw ( px, py, pz )

input:

DOUBLE PRECISION px, py, pz 3-momentum components

The subroutine is used to enter input data, as an alternative to add_particle. It must be called between event_setup_begin and event_setup_end. Each call adds a particle (=detector cell) to the event. The parameters px, py, pz are 3-momentum components in the same units as energy in add_particle. The beam axis is in z-direction. The subroutine is useful with output of Monte Carlo event generators. It can be freely mixed with add_particle.

event_setup_end

must be called after all input particles are entered and before the jet search can be undertaken. No particles can be added to the event afterwards. This subroutine is needed for internal housekeeping. For instance, it provides the proper normalization of the energies of the particles. 


\subsubsection{Setup of initial jet configuration}

\begin{tabular}{lll} 
jets_setup_begin ( njets, Radius ) \\
\hline input: & & \\
INTEGER & njets & number of jets, $N$ \\
DOUBLE PRECISION & Radius & parameter $R$ \\
\hline
\end{tabular}

The subroutine has to be called to begin setup of the initial jet configuration the initial value of the recombination matrix $\left\{z_{a j}\right\}$, necessary for the iterative minimization of $\Omega$, as explained in section 2.2. It is called automatically by Q_search but it must be called explicitly if Q_minimize is used instead. The number of jets, $N$, must be positive. (The event will be reconstructed to the number of jets entered here.) $R$ is the parameter in equation (20). It has to be positive and not too close to zero. The bigger $R$ is, the less energy is left outside the jets. New configurations of jets can be set up any number of times for the same event. The value of the seed from which the random number generator will start for this jet configuration is stored at this point. (From this point until the first invocation of anything random, the seed can be reset by set_seed.) If you need only to change $R$ and proceed with minimization starting from the current configuration, use reset_Radius.

\section{set_seed ( seed )}

input:

INTEGER seed

This is to allow variation in random initial configurations of jets in case there are several local minima. It may be called once for a whole sequence of events - each event starts with a seed set up by the internal random number generator. The seed can be read (see get_seed) and used as a key to regenerate the corresponding configuration of jets (i.e. local minimum; so the local minimum is completely determined by its seed). It must be called after jets_setup_begin but cannot be called after the first invocation of init_z_random or init_random_all or jets_setup_end and until the next jets_setup_begin.

reset_Radius ( Radius )

input:

DOUBLE PRECISION Radius parameter $R$

The subroutine changes the value of the parameter $R$ in equation (20). $R$ has to be positive and not too close to zero. A large value of $R$ means less energy is left outside jets. The subroutine can be called at any time - the current configuration of jets is not affected (only $\Omega$ is recalculated properly). This may 
be useful for setting up interesting variations of the algorithm ("annealing") in which one starts from some small value of $R$ and then changes it gradually, fine-tuning the resulting jet configurations by calls to Q_minimize. With infinitesimal values of $R$, the global minimum occurs for jet configurations with the most energetic particles playing the role of jets, so this can be used to obtain the most energetic (narrow clusters of) particles.

init_z_random_all

The subroutine can only be called between jets_setup_begin and jets_setup_end. It is the simplest way to initialize the recombination matrix $\left\{z_{a j}\right\}$ : completely and uniformly random in the direct product of all the simplices corresponding to particles. If only specific particles need to be randomized, init_z_random( a ) should be used. If only specific particles need to be set non-randomly, init_z ( a, z_in) or assign_to_jet ( $a, j$ ) should be called for those particles. Then init_z_random_all can be called to randomize the remaining particles. If this is not called explicitly, the particles not explicitly initialized are set to "neutral" positions (democratically shared between all jets and the soft energy).

assign_to_jet $(a, j)$

input:

INTEGER a index of the particle

INTEGER $j$ index of the jet

The subroutine can only be called between jets_setup_begin and jets_setup_end. It can be used to set the initial configuration of jets explicitly, for instance, when the output of another jet algorithm is to be fine-tuned. It sets the value $z_{a j}=1$ for the given $a, j$, i.e. directly assigns the $a$-th particle to the $j$-th jet. It must have $1 \leq a \leq n$ and $0 \leq j \leq N ; j=0$ corresponds to soft energy. The subroutine only sets the initial configuration. No elements of the recombination matrix are protected from being changed by subsequent minimizations.

init_z_from ( $a, z_{-}$in $)$

input:

\begin{tabular}{lll} 
INTEGER & $\mathrm{a}$ & index of the particle \\
DOUBLE PRECISION & $z_{-}$in $(0:$ njets_max $)$ & components $z_{a 0}, z_{a 1}, \ldots, z_{a N}$ \\
\hline
\end{tabular}

The subroutine can only be called between jets_setup_begin and

jets_setup_end. It can be used to set the initial configuration of jets explicitly, for instance, to fine-tune the output of another jet algorithm. It initializes the 
recombination matrix $\left\{z_{a j}\right\}$ for the $a$-th particle, i.e. sets $z_{a 0}, z_{a 1}, \ldots, z_{a N}$. Only $N+1$ components of the vector $z_{-}$in $\left(0: n_{j}\right.$ ets_max $)$ are used. The components must be all non-negative but do not need to be normalized correctly - correct normalization will be imposed automatically; z_in(0) is the particle's fraction relegated to soft energy. For instance, $z_{-} i n(j)$ can be some measure of distance between the $a$-th particle and the $j$-th jet from another jet algorithm. The subroutine only sets the initial configuration. No elements of the recombination matrix are protected from being changed by subsequent minimizations.

init_z_random ( a )

input:

INTEGER a index of the particle

The subroutine can only be called between jets_setup_begin and

jets_setup_end. It does random initialization of the recombination matrix $\left\{z_{a j}\right\}$ for the $a$-th particle.

jets_setup_end

The subroutine must be called prior to minimization. It does housekeeping such as initialization of the particles whose recombination matrix elements have not been explicitly initialized by calls from init_z_random.

\subsubsection{Setting algorithm control parameters}

set_maxiter ( maxiter)

input:

INTEGER maxiter maximal number of iterations

The subroutine can be called to change the maximal number of iteration, see 3.4. Default value of the maximal number of iterations is set to 1000 which corresponds to $\sim 1$ second of computing time on a modest PC. It can be called at any time.

set_njets_limits ( nstart, nstop )

input:

INTEGER nstart starting number of jets

INTEGER nstop maximal number of jets

The subroutine is needed in conjunction with Q_search only. It sets the starting and the final number of jets in Q_search (see the end of section 2.2 and description of Q_search in section 3.5.7). The parameters must obey 
$1 \leq$ nstart $\leq$ nstop and nstop $\leq$ njets_max (constant njets_max, set in ojf_par.fh, defines the dimension of matrices and is the maximal allowed number of jets). The default values are: $n$ start $=1$ and nstop $=$ njets_max $=20$. The subroutine can be called any time.

set_ntries ( $\mathrm{n}$ )

input:

INTEGER $\mathrm{n}$ number of tries

The subroutine is needed in conjunction with Q_search only. It sets the number of tries to find the minimum with different random initial configurations for each number of jets (see the end of section 2.2 and description of Q_search, section 3.5.7). The parameter $\mathrm{n}$ must be positive. The larger $\mathrm{n}$, the higher the probability that the found configuration is the global minimum. Note that number of local minima correlates positively with number of hard partons. Usually values $\sim 10$ should suffice. The subroutine can be called at any time.

set_trace_nmoved ( bool)

input:

LOGICAL bool see text

The subroutine with parameter bool=. TRUE. turns on the option in which Q_minimize prints how many particles were moved at each iteration; with bool=.FALSE. it switches the option off (default). The subroutine can be called any time.

\subsubsection{Access to parameters}

get_kinematics ( kinematics )

output:

INTEGER kinematics type of kinematics

The subroutine returns the type of kinematics. The possible values are 1 (spherical kinematics, center of mass collisions) and 2 (cylindrical kinematics, hadron collisions), which is equivalent to constants sphere and cylinder if the header file ojf_kin.fh is included (see also section 3.5.1). The subroutine cannot be called prior to the very first call of event_setup_begin. 
get_nparts ( nparts, e_scale )

output:

INTEGER nparts number of particles in the event

DOUBLE PRECISION e_scale total energy of the event

The subroutine returns the number of particles, $n$, and the total energy in the event. The total energy is the sum of the usual energies of the particles for spherical kinematics $\sum_{a=1}^{n} E_{a}$ and the sum of transverse energies for cylindrical kinematics $\sum_{a=1}^{n} E_{a}^{\perp}$ in in physical units, i.e. prior to the normalization $\sum_{a=1}^{n} E_{a}=1$ or $\sum_{a=1}^{n} E_{a}^{\perp}=1$. In other words, e_scale is the normalization constant. Energy/momentum parameters returned by some other subroutines are normalized by the value of e_scale. The subroutine cannot be called between event_setup_begin and event_setup_end.

get_particle ( a, e, xta, phi, p, ephys, pphys )

input:

\begin{tabular}{lll} 
INTEGER & a & index of the particle \\
\hline output: & & \\
DOUBLE PRECISION & e & normalized energy $E_{a}$ or $E_{a}^{\perp}$ \\
DOUBLE PRECISION & xta & angle $\theta_{a}$ or pseudorapidity $\eta_{a}$ \\
DOUBLE PRECISION & phi & angle $\phi_{a}$ \\
DOUBLE PRECISION & $\mathrm{p}(0: 3)$ & normalized 4 -momentum $p_{a}$ \\
DOUBLE PRECISION & ephys & energy $E_{a}$ or $E_{a}^{\perp}$ not normalized \\
DOUBLE PRECISION & pphys $(0: 3)$ & 4 -momentum $p_{a}$ not normalized
\end{tabular}

The subroutine returns parameters of the $a$-th particle. For spherical kinematics the parameters are the usual energy, $E_{a}$, and the standard angles $\theta_{a}$ (from the beam axis) and $\phi_{a}$. For cylindrical kinematics the parameters are the transverse energy, $E_{a}^{\perp}$, pseudorapidity, $\eta_{a}$, and the angle $\phi_{a}$. The value of $e$ is normalized and the ephys is in the same units as used in input, i.e. ephys $=$ e e escale (see the previous subroutine for e_scale). All angles are in degrees. In both kinematics, $\mathrm{p}(0: 3)$ and pphys $(0: 3)$ are the normalized and non-normalized 4-momenta, $p_{a}$, of the particle. The subroutine cannot be called between event_setup_begin and event_setup_end.

get_njets ( njets )

output:

INTEGER njets number of jets 
The subroutine returns the number of jets in the current configuration of jets. It cannot be called before the first configuration of jets is setup.

get_seed ( seed )

input:

INTEGER seed seed for random generator

The subroutine returns the value of the seed for the random generator, used for setting up the current random jet configuration. The value of the seed is "locked" (causing attempts to reset it to result in program termination) by the first invocation of anything "random" and retained until "unlocked" and reset by jets_setup_begin.

get_Radius ( $\mathrm{R}$ )

output:

DOUBLE PRECISION $\mathrm{R}$ parameter $R$ in eq. (20)

The subroutine returns current value of the parameter $R$ in equation (20).

get_maxiter ( maxiter)

output:

INTEGER maxiter maximal number of iteration

The subroutine returns the maximal number of iterations (see section 3.4).

get_njets_limits ( nstart, nstop )

output:

INTEGER nstart starting number of jets

INTEGER nstop maximal number of jets

The subroutine returns the current values of the starting number of jets and the maximal number of jets in the subroutine Q_search (see the end of section 2.2 and description of Q_search in section 3.5.7).

get_ntries $(\mathrm{n})$

output:

INTEGER $\mathrm{n}$ number of tries

The subroutine returns the current number of tries in Q_search, the number 
of attempts to find minimum with different random initial configurations for each number of jets (see the end of section 2.2 and description of Q_search, section 3.5.7).

\subsubsection{Access to results}

\begin{tabular}{|c|c|c|}
\hline \multicolumn{3}{|l|}{ output: } \\
\hline DOUBLE PRECISION & omega & value of $\Omega$ \\
\hline DOUBLE PRECISION & $\mathrm{y}$ & value of $Y$ \\
\hline DOUBLE PRECISION & esoft & value of $E_{\mathrm{s}}$ \\
\hline
\end{tabular}

The subroutine returns the value of $\Omega, Y$ and $E_{\text {soft }}$. Whenever a jet configuration is set up or modified, the corresponding values of $\Omega, Y$ and $E_{\text {soft }}$ are recalculated and can be retrieved using this subroutine.

\begin{tabular}{|c|c|c|}
\hline INTEGER & j & index of the jet \\
\hline \multicolumn{3}{|l|}{ output: } \\
\hline \multirow[t]{2}{*}{ DOUBLE PRECISION } & e & normalized energy \\
\hline & & or normalized transverse energy \\
\hline DOUBLE PRECISION & xta & angle $\theta_{j}$ or pseudorapidity $\eta_{j}$ \\
\hline DOUBLE PRECISION & phi & angle $\phi_{j}$ \\
\hline DOUBLE PRECISION & $q(0: 3)$ & normalized 4-momentum $q_{j}$ \\
\hline DOUBLE PRECISION & qtilde $(0: 3)$ & 4-direction $\tilde{q}_{j}$ \\
\hline \multirow[t]{2}{*}{ DOUBLE PRECISION } & ephys & energy (or transverse energy) \\
\hline & & in physical units \\
\hline DOUBLE PRECISION & qphys $(0: 3)$ & 4-momentum $q_{j}$ in physical units \\
\hline
\end{tabular}

The subroutine returns parameters of the $j$-th jet, where $j$ obeys $0 \leq j \leq N$ and $j=0$ is the zeroth "jet", name for the fractions of particles that do not belong to any jet (i.e. soft energy). For spherical kinematics the parameters are the usual energy, $E_{j}$, normalized e and non-normalized ephys (i.e. in the units of energy used in the input), the standard angles $\theta_{j}$ (from the beam axis) and $\phi_{j}$. For cylindrical kinematics the parameters are the transverse energy, $E_{j}^{\perp}$, normalized e and non-normalized ephys (i.e. in the units of energy used 
in the input), pseudorapidity $\eta_{j}$ and the standard angle $\phi_{j}$. All angles are in degrees. For both kinematics the parameters $q(0: 3)$ and qtilde $(0: 3)$ are the normalized and non-normalized 4 -momentum of the jet. $\tilde{q}_{j}$ is the 4 -direction defined in section 2.1 .

get_z ( a, z_out )

input:

\begin{tabular}{lll} 
INTEGER & a & index of the particle \\
\hline
\end{tabular}

output:

DOUBLE PRECISION z_out(0:njets_max) components $z_{a 0}, z_{a 1}, \ldots, z_{a N}$

The subroutine returns the components $z_{a 0}, z_{a 1}, \ldots, z_{a N}$ of the recombination matrix for the $a$-th particle. $a$ must satisfy $1 \leq a \leq n$. The value of $z_{-}$out $(j)$ is the $a$-th particle contribution to the $j$-jet and $j=0$ corresponds to the soft energy. Note: $\sum_{j=0}^{N} z_{-}$out $(j)=1$.

\begin{tabular}{|c|c|c|}
\hline \multicolumn{3}{|l|}{ input: } \\
\hline INTEGER & a & index of the particle \\
\hline \multicolumn{3}{|l|}{ output: } \\
\hline \multirow[t]{2}{*}{ INTEGER } & total_jets & number of jets the particle \\
\hline & & belongs to \\
\hline INTEGER & jet $\left(0: n j e t s \_m a x\right)$ & indices of the jets \\
\hline DOUBLE PRECISION & $z j(0: n j$ ets_max $)$ & corresponding $z_{a j}$ \\
\hline
\end{tabular}

For the $a$-th particle the subroutine returns: the number of jets (including soft energy 0-th "jet") which include a non-zero fraction of the particle $\left(z_{a j} \neq 0\right)$, the labels of the jets and the corresponding values of $z_{a j}$ in such an order that $z j(k) \geq z j(j+1)$. In other words, the vector $z j\left(0: n j e t s \_m a x\right)$ is the collection of $z_{a 0}, z_{a 1}, \ldots, z_{a N}$ ordered by their value (descending from the left to right); only the components (0:total_jets-1) are different from zero. 


\begin{tabular}{|c|c|c|}
\hline \multicolumn{3}{|l|}{ input: } \\
\hline INTEGER & j & index of the jet \\
\hline \multicolumn{3}{|l|}{ output: } \\
\hline \multirow[t]{2}{*}{ INTEGER } & nwhole & number of particles entirely \\
\hline & & belonging to the jet \\
\hline INTEGER & whole_a (0:nparts_max $)$ & labels of the particles \\
\hline \multirow[t]{2}{*}{ INTEGER } & nfract & number of particles \\
\hline & & belonging in some fraction \\
\hline INTEGER & fract_a $(0:$ nparts_max $)$ & labels of the particles \\
\hline DOUBLE PRECISION & fract_z $(0:$ nparts_max $)$ & the fraction $z_{a j}$ \\
\hline
\end{tabular}

For the $j$-th jet ( $0 \leq j \leq N, j=0$ is the soft energy) the subroutine returns:

- number of the particles wholly in the jet, i.e. $z_{a j}=1$

- vector whole_a (0:nparts_max) with labels of such particles (indices $a$ )

- number of particles partially in the jet, i.e $0<z_{a j}<1$

- vector fract_a(0:nparts_max) with labels of such particles (indices $a$ )

- vector fract_z(0:nparts_max) with corresponding $z_{a j}$ for such particles.

The latter two vectors are synchronously ordered so that subsequent components of fract_z $(0:$ nparts_max $)$ do not increase.

\subsubsection{Sample print routines}

print_z_raw

is an example subroutine to print the recombination matrix $\left\{z_{a j}\right\}$. A possible output may look like:

\begin{tabular}{|c|c|c|c|c|}
\hline a & background & 1 & 2 & 3 \\
\hline & & & & \\
\hline 1 & 0.0000 & 0.0000 & 0.0000 & 1.0000 \\
\hline 2 & 0.0000 & 0.0000 & 0.0000 & 1.0000 \\
\hline 3 & 0.0000 & 0.0000 & 0.0000 & 1.0000 \\
\hline 4 & 0.0000 & 0.0000 & 0.0000 & 1.0000 \\
\hline
\end{tabular}

print_z_nice

is an example subroutine to print the recombination matrix $\left\{z_{a j}\right\}$. A possible 
output may look like:

recombination matrix $\mathrm{z}$ by particle label a:

\begin{tabular}{|c|c|c|c|c|}
\hline a & background & 1 & $2^{u}$ & 3 \\
\hline--- & ----- & -- & -- & 1 \\
\hline 1 & - & - & - & 1 \\
\hline 2 & - & - & - & 1 \\
\hline 3 & - & - & - & 1 \\
\hline 4 & - & - & - & 1 \\
\hline
\end{tabular}

print_jets

is an example subroutine to print properties of jets. See the output of the example program in section 3.7.2.

print_particles

is an example subroutine to print properties of particles. A possible output may look like:

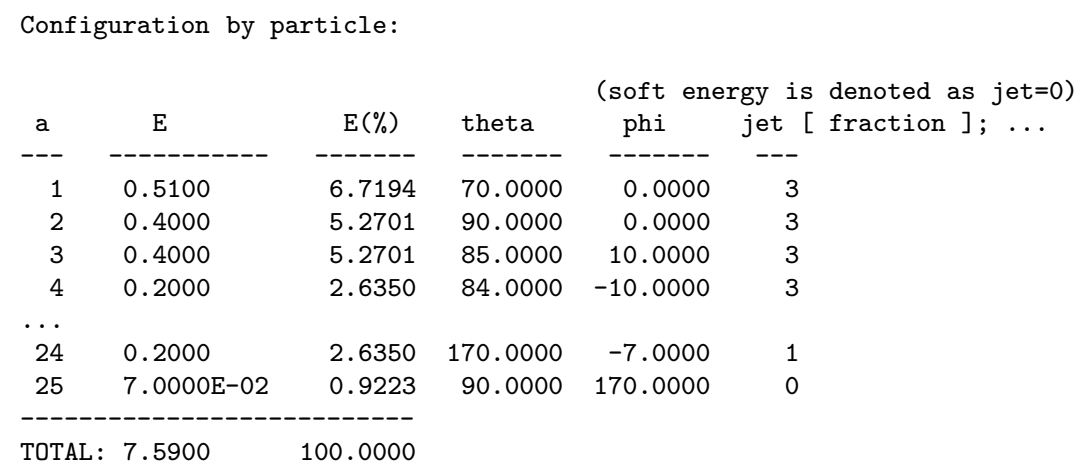

\subsubsection{Example subroutine of straightforward jet search Q_search}

This is a simple jet search subroutine using Q_minimize as a key component. It is possible that the user may want to modify it, for example, when trying to do something with local minima. This subroutine uses only interface routines; it does not access internal data.

The subroutine tries to find the configuration of jets which minimizes $\Omega$ and ensures that $\Omega<\omega_{\text {cut }}$ with the minimal number of jets (njets) starting from 
the number of jets previously set via set_njets_start (usually the same for all events). For each number of jets, the search is repeated ntries times, each time with a different random initial value of the recombination matrix $\left\{z_{a j}\right\}$ and the configuration with the lowest value of $\Omega$ is retained as a result. Failure of the search is signaled by the condition njets $=0$.

Note that Q_search randomizes the initial value of $\left\{z_{a j}\right\}$, so it is meaningless to use it if one wants to specify the initial configuration for $\left\{z_{a j}\right\}$. In this case, the user should use Q_minimize directly. We comment that some other control options could be to continue attempts until a specified number of attempts fails to yield a better configuration or to stop the search for new minimum if, for example, the first three random initial configurations yielded the same configurations (the event has a single local minimum which is automatically the global one; this is quite likely and may be useful if CPU time is an issue).

\subsection{Compilation}

Optimal Jet Finder consists of the following files:

- ojf_014.f main file contains all subroutines and functions

- ojf_com.fh contains definitions of common blocks

- ojf_par.fh contains definitions of parameters

- ojf_kin.fh contains definition of kinematics type parameters

The example programs example.f, ww160.f, ww160a.f with input or output files example.in, ww160.in, ww160.out, ww160a.out are added.

To compile and run any of example programs with OJF under Linux equipped with g77 the user can type:

$$
\begin{array}{ll}
\text { g77 user_program.f ojf_014.f -o executable_file } & \text { (enter) } \\
\text { executable_file } & \text { (enter) }
\end{array}
$$

where user_program.f is the name of the user own program applying OJF. Each example program example.f, ww160.f or ww160a.f can be used in its place. The files ojf_com.fh, ojf_par.fh, and ojf_kin.fh should be available in the current directory (but not compiled).

\subsection{Example}

The simplest possible example, file example.f below, should give the idea how Optimal Jet Finder can be used. The file example.in contains input data. 
Each line corresponds to one particle and consists of $E_{a}, \theta_{a}$ and $\phi_{a}$ for that particle. The user is encouraged to study subroutine Q_search and programs ww160.f, ww160a.f providing additional, more advanced examples.

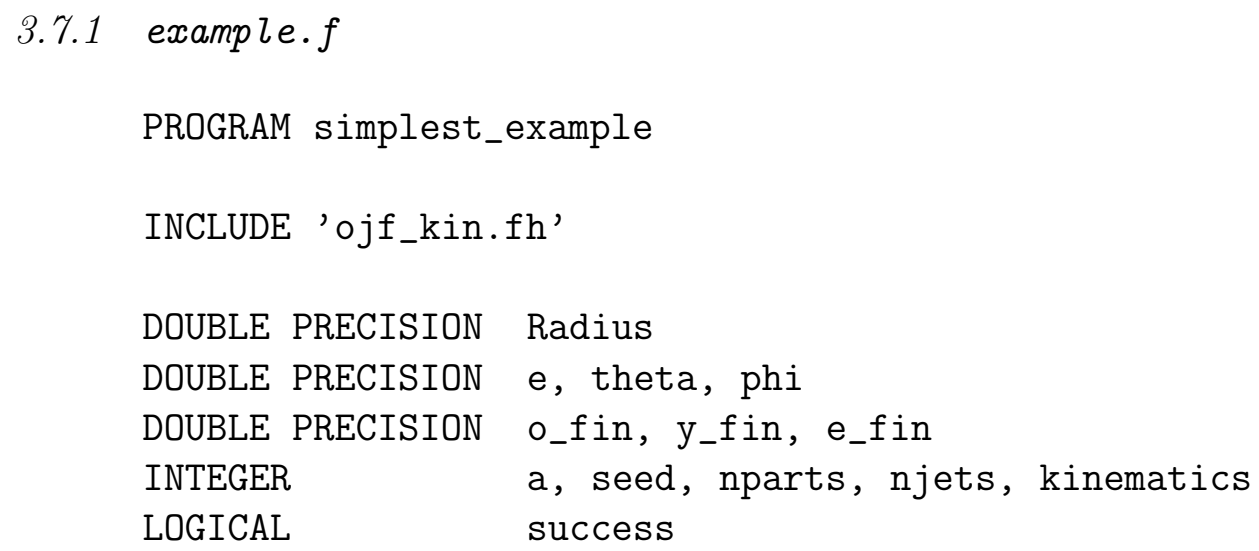

number of jets is chosen

njets $=3$

seed for random generation of the recombination matrix

seed $=13$

$R$ parameter from equation (20), section 2.2

Radius $=1.0$

choose spherical (lepton collisions) kinematics

kinematics $=$ sphere

file with input data is opened

$\operatorname{OPEN}(10$, FILE=' example.in', FORM='formatted', STATUS = 'old')

input event setup starts

CALL event_setup_begin ( kinematics )

loop over all particles in the event

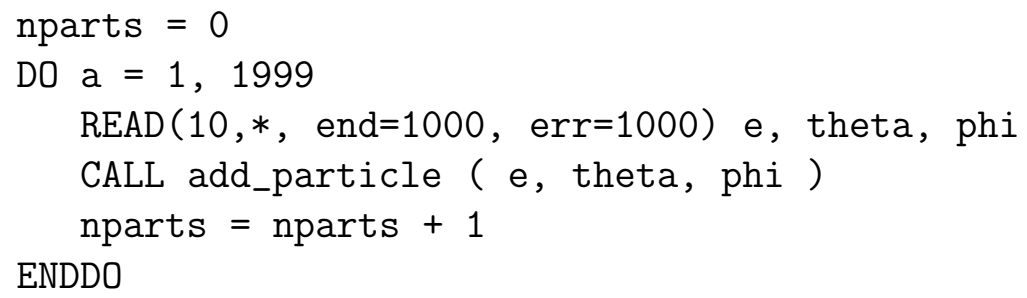


set up random the initial value of the recombination matrix

CALL jets_setup_begin ( njets, Radius )

CALL set_seed ( seed )

CALL init_z_random_all

CALL jets_setup_end

minimize $\Omega$

CALL Q_minimize ( success )

IF (.NOT. success) STOP 'minimum not found'

get and print the values of $\Omega, Y$ and $E_{\text {soft }}$ for the final jet configuration

CALL get_criterion ( o_fin, y_fin, e_fin )

$$
\begin{aligned}
& \operatorname{WRITE}(*, *) \text { 'Omega }=\text { ', o_fin } \\
& \operatorname{WRITE}(*, *) \text { 'Y } \quad=\text {, y_fin } \\
& \operatorname{WRITE}(*, *) \text { 'E_soft }=\text { ', e_fin }
\end{aligned}
$$

prints properties of the resulting jets

call print_jets

END

\subsubsection{Output of the example}

$$
\begin{array}{ll}
\text { Omega } & =0.293404849 \\
\mathrm{Y} & =0.0338528071 \\
\text { E_soft } & =0.259552042 \\
\text { SPHERE: } & 3 \text { jets processed }
\end{array}
$$

Configuration by jet:

\begin{tabular}{rccrr} 
jet & $E$ & $E(\%)$ & theta & \multicolumn{1}{c}{ phi } \\
--- & -------- & ------ & ------ & ------ \\
1 & 1.380 & 18.1818 & 138.3848 & -52.8876 \\
2 & 1.220 & 16.0738 & 124.4338 & -26.6115 \\
3 & 3.020 & 39.7892 & 81.0226 & 0.3566
\end{tabular}

TOTAL: $5.6200 \quad 74.0448$

Particle content by jet:

jet label 1 ( 3 particle(s) ): 


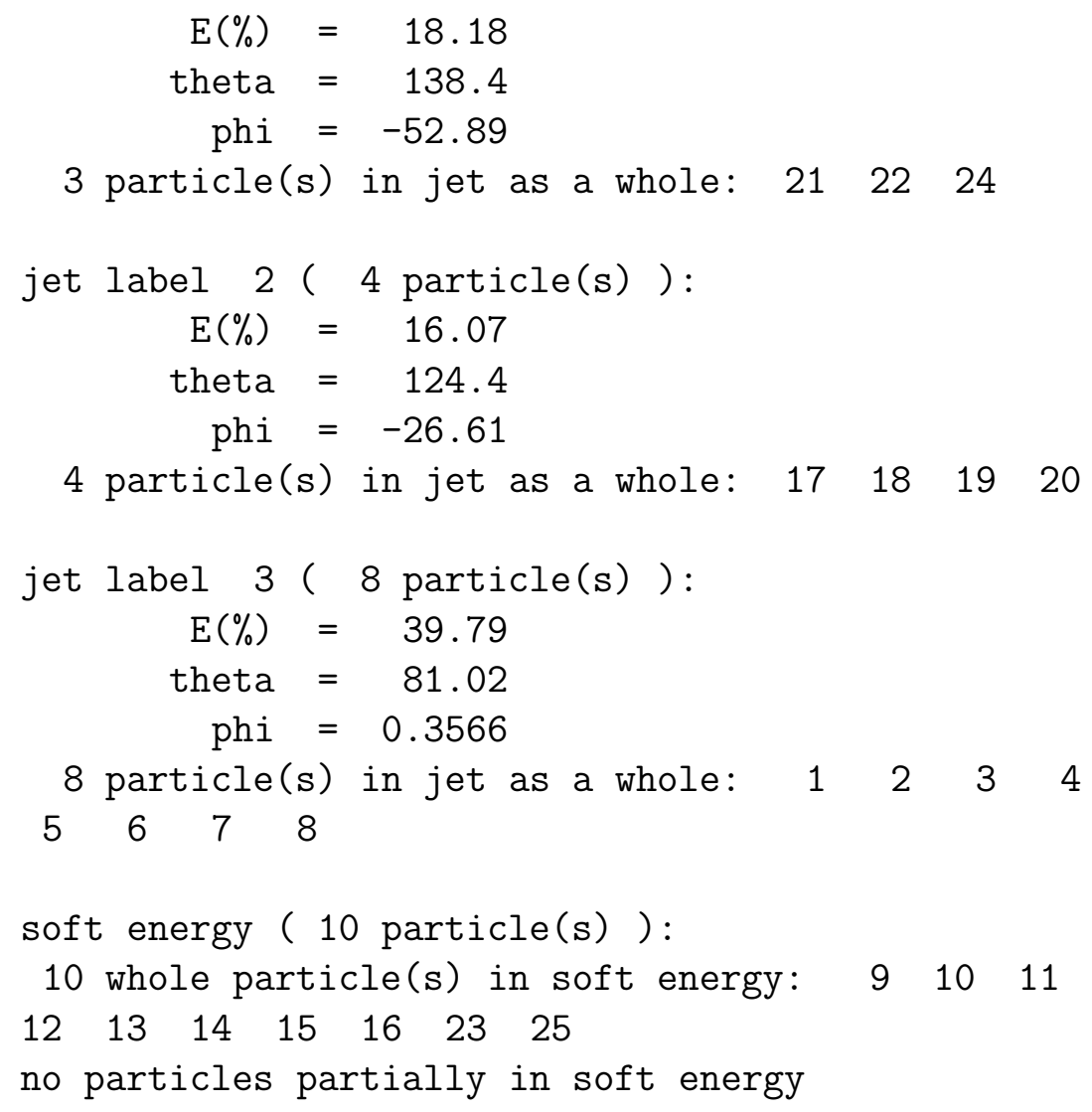

4 Definitions of constants: ojf_par.fh

In this section we explain the meaning of the parameters defined in the header file ojf_par.fh and give their default values.

INTEGER njets_max $=50$

The maximal number of jets; used for example to define the size of matrices.

INTEGER nparts_max $=2000$

The maximal number of particles in the event; used for example to define the size of matrices.

DOUBLE PRECISION zero $=0$

DOUBLE PRECISION one $=1$

DOUBLE PRECISION inf $=10^{100}$

are the numerical constants.

DOUBLE PRECISION eps_snap $=10^{-3}$

If $z_{a j}<$ eps_snap than $z_{a j}$ is set to zero, i.e. the particle is snapped to the 
boundary of the simplex. The parameter is used in subroutines $z_{-}$snap and z_assert.

DOUBLE PRECISION eps_round $=10^{-6}$

DOUBLE PRECISION eps_sum $=10^{-8}$

DOUBLE PRECISION eps_sum0 $=10^{-6}$

DOUBLE PRECISION eps_sum $1=10^{-4}$

The constants are used to keep control of rounding errors. If some variable exceeds the allowing range of values more than eps, the error message is generated and the program is terminated. The constants are used in the subroutines d_minus_snap, z_snap, d_assert, z_assert, z_force_to_simplex and in the function pos_prod.

DOUBLE PRECISION eps_norm $=10^{-6}$

The constant is used to determine whether the norm of the 3-vector $\mathbf{q}_{j}$ (or transverse part of the norm in case of cylindrical dynamics) is zero. It is used in the subroutine j_eval_nonlinear.

DOUBLE PRECISION eps_Et $=10^{-6}$

The constant is used to handle small values of the transverse energy of a jet. It is used in the subroutines grad_Y and j_eval_nonlinear.

DOUBLE PRECISION eps_dist $=10^{-6}$

See section 3.4. The constant is used to determine when to stop subsequent reductions of the step $\tau$. The constant is used in the subroutine Q_minimize_wrt.

DOUBLE PRECISION eps_radius $=10^{-3}$

The constant sets the limit on the smallest value of $R$, the parameter from equation (20). It is used in the subroutines jet_setup_begin and reset_Radius.

DOUBLE PRECISION inf_step $=10^{30}$

See section 3.4. "Infinite" step means that the particle should not be moved. The constant is used in subroutines Q_minimize_wrt, d_eval_step and

z_move_by.

It is imaginable that some of the parameters above may need to be changed but the user is advised to be careful when doing this. In particular, smaller values of some parameters would enhance sensitivity to rounding errors, causing the safety checks to generate error messages and terminate the program. One may change eps_snap to a smaller value, say $10^{-5}$, and see if the results would change; for a small fraction of events this may slow the finding of jets but help to better identify local minima.

INTEGER random_m $=259200$

The constant is used by the random number generator, the subroutine seed 
and the function random().

The constants below play a technical role and are not supposed to be changed. The reason for defining them is cleared in the next section.

INTEGER par_Et $=4$

INTEGER par_eta $=5$

INTEGER par_Eteta $=6$

INTEGER par_y $=7$

INTEGER par_p0shmpzch $=8$

INTEGER par_tilde $=9$

\section{Common block definitions: ojf_com.fh}

The header file ojf_com.fh contains common block definitions and is included in most of the subroutines. The user is not supposed to write to common blocks directly but to use interface subroutines. Data that cannot be accessed that way is not supposed to be used by the user.

\subsection{Input of the event}

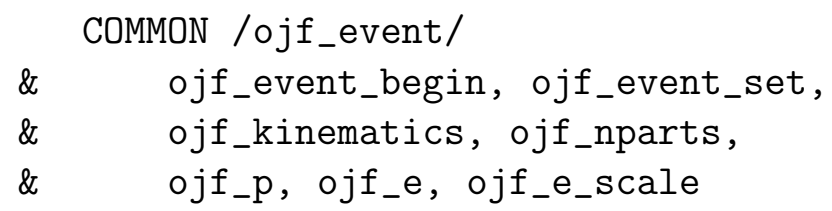

LOGICAL ojf_event_begin, ojf_event_set

The two logical values bracket the event setup:

FALSE, FALSE - at start of program, no event has been set up;

TRUE , FALSE - event setup in progress, adding particles;

FALSE, TRUE - event setup completed, can search for jets.

INTEGER ojf_kinematics

The variable marks the type of kinematics: 1 - spherical kinematics of lepton collisions, 2 - cylindrical kinematics of hadron collisions.

INTEGER ojf_nparts

The number of particles in the event. 
DOUBLE PRECISION ojf_p $(0: 6,1:$ nparts_max $)$

The matrix stores the properties of particles:

ojf_p(0, particle_label) energy $E_{a}$

ojf_p(1, particle_label) x-component of momentum $p_{a}$

ojf_p(2, particle_label) y-component of momentum $p_{a}$

ojf_p(3, particle_label) z-component of momentum $p_{a}$

ojf_p (4, particle_label) transverse energy $E_{a}^{\perp}$

ojf_p(5, particle_label) pseudorapidity $\eta_{a}$

ojf_p (6, particle_label) combination $E_{a}^{\perp} \cdot \eta_{a}$

and particle_label is the index $a$ of the particle. The constants par_Et $=4$, par_eta $=5$, par_Eteta $=6$ are defined to access the components of the matrix, e.g. ojf_p(par_Eteta, particle_label).

DOUBLE PRECISION ojf_e(1:nparts_max)

The vector stores the energies of the particles.

DOUBLE PRECISION ojf_e_scale

The variable stores the energy scaling factor (see section 3.2).

\subsection{Configuration of jets (output)}

$\begin{array}{ll} & \text { COMMON /ojf_jets/ } \\ \& & \text { ojf_jets_begin, ojf_jets_set, } \\ \& & \text { ojf_njets, ojf_seed, ojf_Radius, } \\ \& & \text { ojf_z, ojf_b, ojf_q, } \\ \& & \text { ojf_Omega, ojf_Y, ojf_Esoft }\end{array}$

LOGICAL ojf_jets_begin, ojf_jets_set

The two logical values bracket setup of initial jet configuration:

FALSE, FALSE - at start of program, or after event set up;

TRUE, FALSE - jets setup in progress, change anything;

FALSE, TRUE - jets setup complete, can do minimization.

INTEGER ojf_njets

The number of jets in the current configuration.

INTEGER ojf_seed

The seed used to generate the current (random) jet configuration.

DOUBLE PRECISION ojf_Radius 
The value of $R$, the parameter in equation (20).

DOUBLE PRECISION ojf_z $(0: n j$ jets_max, 1 :nparts_max $)$

The recombination matrix, $\left\{z_{a j}\right\}$.

LOGICAL ojf_b(0:njets_max, 1 :nparts_max)

It is used to indicate that the particle belongs to (TRUE) or does not belong (FALSE) to the boundaries of the simplex, i.e. $z_{a j}=0$.

DOUBLE PRECISION ojf_q $(0: 12,1:$ jets_max $)$

The matrix stores the properties of particles:

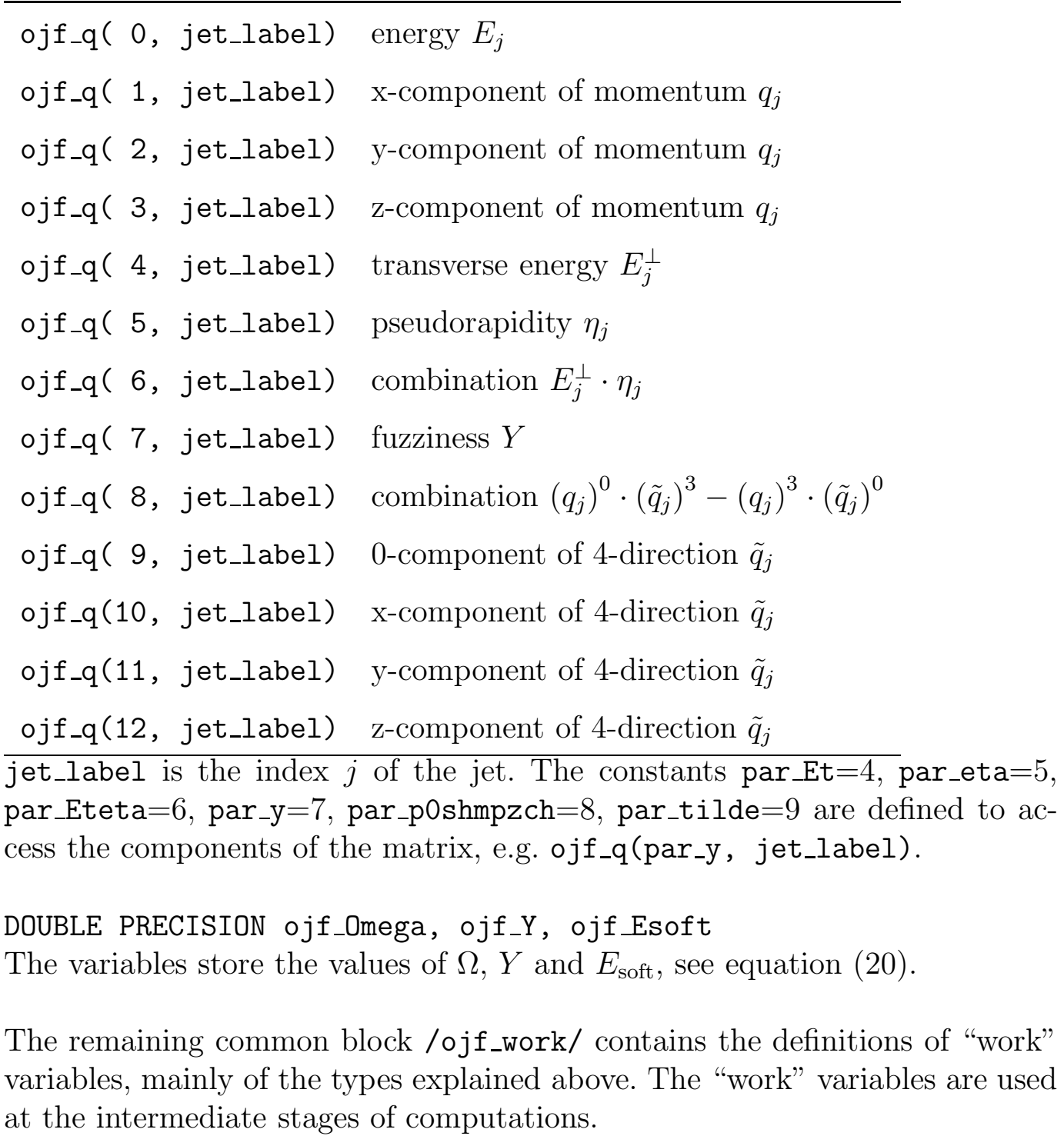




\section{Conclusion}

We implemented an algorithm for minimization of $\Omega$, the criterion for finding the optimal jet configuration. The user is provided with the example program and subroutine Q_search as the simplest possible scenarios of jet finding. It is not inconceivable that practical applications will require more sofisticated ways of using the provided library. To facilitate this, we supplied the user with numerous interface subroutines allowing for easy control of the program.

We did not explore all possible ideas for optimizing of jet search as we wanted to leave the program generic. For example, in some cases it is possible to apply OJF in a few stages. First, apply it to particles with the highest energies, then use the result as the initial value of the recombination matrix for the next stage at which the rest of particles are added (or some next portion). It may increase the speed of finding jets without significant deterioration of the results [1]. However this depends on the particular physical process at hand.

\section{References}

[1] D. Yu. Grigoriev, E. Jankowski, F. V. Tkachov, e-print hep-ph/0301185, to be published.

[2] R. Barlow, Rep. Prog. Phys. 36, 1067 (1993).

[3] F. V. Tkachov, Phys. Rev. Lett. 73, 2405 (1994); Erratum, 74, 2618 (1995).

[4] F. V. Tkachov, Int. J. Mod. Phys. A12, 5411 (1997).

[5] F. V. Tkachov, Int. J. Mod. Phys. A17, 2783 (2002).

[6] D. Yu. Grigoriev and F. V. Tkachov, e-print hep-ph/9912415.

[7] F. V. Tkachov, Part. Nucl., Letters 2[111], 28 (2002).

[8] N. Amos et al., contribution to CHEP95, URL: http://www.hep.net/chep95/html/papers/p155/.

[9] P. C. Bhat, H. Prosper, and S. S. Snyder, Int. J. Mod. Phys. A13, 5113 (1998).

[10] C. F. Berger et al., e-Print hep-ph/0202207.

[11] The FORTRAN code ver. OJF_014 is currently available from http://www.inr.ac.ru/ ftkachov/projects/jets/

[12] http://www.oberon.ch.

[13] F. V. Tkachov, e-print hep-ph/0202033. 
[14] The first realistic test was run by P. Achard (L3, CERN) in 1999 with a sample of about $10^{5}$ events.

[15] F. V. Tkachov, e-print hep-ph/0111035.

[16] S. Catani et al., Phys. Lett. 269B, 432 (1991).

[17] http://hepwww.rl.ac.uk/theory/seymour/ktclus/ 